\title{
Microplastics as contaminants in the marine environment: a review
}

Matthew Cole ${ }^{1^{*}}$, Pennie Lindeque ${ }^{1}$, Claudia Halsband ${ }^{2} \&$ Tamara Galloway $^{3}$

${ }^{1}$ Plymouth Marine Laboratory, Prospect Place, The Hoe, Plymouth, PL1 3DH, UK.

2 Akvaplan-niva AS, FRAM - High North Research Centre for Climate and the Environment, N-9296 Tromsø, Norway.

${ }^{3}$ College of Life and Environmental Sciences: Biosciences, Hatherly Laboratories, University of Exeter, Prince of Wales Road, Exeter, EX4 4PS, UK.

* Corresponding author: phone: +44 (0)1752 633450; fax: +44 (0)1752 633101; email: mcol@pml.ac.uk.

\section{Abstract}

Since the mass production of plastics began in the 1940s, microplastic contamination of the marine environment has been a growing problem. Here, a review of the literature has been conducted with the following objectives: (1) to summarise the properties, nomenclature and sources of microplastics; (2) to discuss the routes by which microplastics enter the marine environment; (3) to evaluate the methods by which microplastics are detected in the marine environment; (4) to assess spatial and temporal trends of microplastic abundance; and (5) to discuss the environmental impact of microplastics. Microplastics are both abundant and widespread within the marine environment, found in their highest concentrations along coastlines and within mid-ocean gyres. Ingestion of microplastics has been demonstrated in a range of marine organisms, a process which may facilitate the transfer of chemical additives or hydrophobic waterborne pollutants to biota. We conclude by highlighting key future research areas for scientists and policymakers. 


\section{Keywords}

Microplastics; marine litter; plastic debris; priority pollutant.

\section{Introduction}

Plastics are synthetic organic polymers, which are derived from the polymerisation of monomers extracted from oil or gas (Derraik, 2002; Rios et al., 2007; Thompson et al., 2009b). Since the development of the first modern plastic, 'Bakelite', in 1907, a number of inexpensive manufacturing techniques have been optimised, resulting in the mass production of a plethora of lightweight, durable, inert and corrosionresistant plastics (PlasticsEurope, 2010). These attributes have led to the extensive use of plastics in near inexhaustible applications (Andrady, 2011). Since mass production began in the 1940s, the amount of plastic being manufactured has increased rapidly, with 230 million tonnes of plastic being produced globally in 2009 (PlasticsEurope, 2010), accounting for $\sim 8 \%$ of global oil production (Thompson et al., 2009b).

Whilst the societal benefits of plastic are far-reaching (Andrady and Neal, 2009), this valuable commodity has been the subject of increasing environmental concern. Primarily, the durability of plastic that makes it such an attractive material to use also makes it highly resistant to degradation, thus disposing of plastic waste is problematic (Barnes et al., 2009; Sivan, 2011). Exacerbated by the copious use of throw-away "user" plastics (e.g. packaging material), the proportion of plastic contributing to municipal waste constitutes $10 \%$ of waste generated worldwide (Barnes et al., 2009). While some plastic waste is recycled, the majority ends up in landfill where it may take centuries for such material to breakdown and decompose (Barnes et al., 2009; Moore, 2008). Of particular concern are plastics that, through 
indiscriminate disposal, are entering the marine environment (Gregory, 2009). Despite plastics being an internationally recognised pollutant with legislation in place aimed to curb the amount of plastic debris entering the marine environment (Gregory, 2009; Lozano and Mouat, 2009), Thompson (2006) estimates up to $10 \%$ of plastics produced end up in the oceans, where they may persist and accumulate.

The impact that large plastic debris, known as 'macroplastics', can have on the marine environment has long been the subject of environmental research. The presence of macroplastics in the marine environment presents an aesthetic issue, with economic repercussions for the tourist industry, a hazard for numerous marineindustries (e.g. shipping, fishing, energy production, aquaculture) as plastic may result in entanglement and damage of equipment, and significant environmental concerns (Barnes et al., 2009; Derraik, 2002; Sivan, 2011). The environmental impact of macroplastics include: the injury and death of marine birds, mammals, fish and reptiles resulting from plastic entanglement and ingestion (Derraik, 2002; Gregory, 2009; Lozano and Mouat, 2009); the transport of non-native marine species (e.g. bryozoans) to new habitats on floating plastic debris (Barnes, 2002; Derraik, 2002; Winston, 1982); and the smothering of the seabed, preventing gas-exchange and creating artificial hard-grounds, resulting from sinking plastic debris (Gregory, 2009; Moore, 2008).

In recent years, there has been increasing environmental concern about 'microplastics': tiny plastic granules used as scrubbers in cosmetics and air-blasting, and small plastic fragments derived from the breakdown of macroplastics (Derraik, 2002; Ryan et al., 2009; Thompson et al., 2004). The presence of small plastic fragments in the open ocean was first highlighted in the 1970s (Carpenter and Smith, 1972), and a renewed scientific interest in microplastics over the past decade has revealed that these contaminants are widespread and ubiquitous within the marine 
environment, with the potential to cause harm to biota (Rands et al., 2010; Sutherland et al., 2010). Owing to their small size, microplastics are considered bioavailable to organisms throughout the food-web. Their composition and relatively large surface area make them prone to adhering waterborne organic pollutants and to the leaching of plasticisers that are considered toxic. Ingestion of microplastics may therefore be introducing toxins to the base of the food chain, from where there is potential for bioaccumulation (Teuten et al., 2009).

The objectives of this review are: (1) to summarise the properties, nomenclature and sources of microplastics; (2) to discuss the routes by which microplastics enter the marine environment; (3) to evaluate the methods by which microplastics are detected in the marine environment; (4) to ascertain spatial and temporal trends of microplastic abundance; and (5) to determine the environmental impact of microplastics.

\section{Microplastics}

Whilst macroplastic debris has been the focus of environmental concern for some time, it is only since the turn of the century that tiny plastic fragments, fibres and granules, collectively termed "microplastics", have been considered as a pollutant in their own right (Ryan et al., 2009; Thompson et al., 2004). Microplastics have been attributed with numerous size-ranges, varying from study to study, with diameters of $<10 \mathrm{~mm}$ (Graham and Thompson, 2009), <5 mm (Barnes et al., 2009; Betts, 2008), 2-6 mm (Derraik, 2002), <2 mm (Ryan et al., 2009) and <1 mm (Browne et al., 2007; Browne et al., 2010; Claessens et al., 2011). This inconsistency is particularly problematic when comparing data referring to microplastics, making it increasingly important to create a scientific standard (Claessens et al., 2011; Costa et al., 2010). Recently, Andrady (2011) has suggested adding the term "mesoplastics" to scientific 
nomenclature, to differentiate between small plastics visible to the human eye, and those only discernible with use of microscopy.

\section{Primary microplastics}

Plastics that are manufactured to be of a microscopic size are defined as primary microplastics. These plastics are typically used in facial-cleansers and cosmetics (Zitko and Hanlon, 1991), or as air-blasting media (Gregory, 1996), whilst their use in medicine as vectors for drugs is increasingly reported (Patel et al., 2009). Under the broader size definitions of a microplastic, virgin plastic production pellets (typically 2$5 \mathrm{~mm}$ in diameter) can also be considered as primary microplastics, although their inclusion within this category has been criticised (Andrady, 2011; Costa et al., 2010).

Microplastic "scrubbers", used in exfoliating hand cleansers and facial scrubs, have replaced traditionally used natural ingredients, including ground almonds, oatmeal and pumice (Derraik, 2002; Fendall and Sewell, 2009). Since the patenting of microplastic scrubbers within cosmetics in the 1980s, the use of exfoliating cleansers containing plastics has risen dramatically (Fendall and Sewell, 2009; Zitko and Hanlon, 1991). Typically marketed as "micro-beads" or "micro-exfoliates", these plastics can vary in shape, size and composition depending upon the product (Fendall and Sewell, 2009). For example, Gregory (1996) reported the presence of polyethylene and polypropylene granules $(<5 \mathrm{~mm})$ and polystyrene spheres $(<2 \mathrm{~mm})$ in one cosmetic product. More recently, Fendall and Sewell (2009) reported an abundance of irregularly shaped microplastics, typically $<0.5 \mathrm{~mm}$ in diameter with a mode size $<0.1 \mathrm{~mm}$, in another cosmetic product.

Primary microplastics have also been produced for use in air-blasting technology (Derraik, 2002; Gregory, 1996). This process involves blasting acrylic, melamine or 
polyester microplastic scrubbers at machinery, engines and boat hulls to remove rust and paint (Browne et al., 2007; Derraik, 2002; Gregory, 1996). As these scrubbers are used repeatedly until they diminish in size and their cutting power is lost, they will often become contaminated with heavy metals (e.g. Cadmium, Chromium, Lead) (Derraik, 2002; Gregory, 1996).

\section{Secondary microplastics}

Secondary microplastics describe tiny plastic fragments derived from the breakdown of larger plastic debris, both at sea and on land (Ryan et al., 2009; Thompson et al., 2004). Over time a culmination of physical, biological and chemical processes can reduce the structural integrity of plastic debris, resulting in fragmentation (Browne et al., 2007).

Over prolonged periods, exposure to sunlight can result in photo-degradation of plastics; ultraviolet (UV) radiation in sunlight causes oxidation of the polymer matrix, leading to bond cleavage (Andrady, 2011; Barnes et al., 2009; Browne et al., 2007; Moore, 2008; Rios et al., 2007). Such degradation may result in additives, designed to enhance durability and corrosion resistance, leaching out of the plastics (Talsness et al., 2009). The cold, haline conditions of the marine environment are likely to prohibit this photo-oxidation; plastic debris on beaches, however, have high oxygen availability and direct exposure to sunlight so will degrade rapidly, in time turning brittle, forming cracks and "yellowing" (Andrady, 2011; Barnes et al., 2009; Moore, 2008). With a loss of structural integrity, these plastics are increasingly susceptible to fragmentation resulting from abrasion, wave-action and turbulence (Barnes et al., 2009; Browne et al., 2007). This process is ongoing, with fragments becoming smaller over time until they become microplastic in size (Fendall and Sewell, 2009; Rios et al., 2007; Ryan et al., 2009). It is considered that microplastics might further 
degrade to be nanoplastic in size, although the smallest microparticle reportedly detected in the oceans at present is $1.6 \mu \mathrm{m}$ in diameter (Galgani et al., 2010). The presence of nanoplastics in the marine environment is likely to be of increasing significance in the years to come, and researchers, including Andrady (2011), have already begun to speculate on the impact that such a pollutant might have on the base of the marine food web.

The development of biodegradable plastics is often seen as a viable replacement for traditional plastics. However, they too may be a source of microplastics (Thompson et al., 2004). Biodegradable plastics are typically composites of synthetic polymers and starch, vegetable oils or specialist chemicals (e.g. TDPA ${ }^{\mathrm{TM}}$ ) designed to accelerate degradation times (Derraik, 2002; O'Brine and Thompson, 2010; Ryan et al., 2009; Thompson et al., 2004) that, if disposed of appropriately, will decompose in industrial composting plants under hot, humid and well-aerated conditions (Moore, 2008; Thompson, 2006). However, this decomposition is only partial: whilst the starch components of the bio-plastic will decompose, an abundance of synthetic polymers will be left behind (Andrady, 2011; Roy et al., 2011; Thompson et al., 2004). In the relatively cold marine environment, in the absence of terrestrial microbes, decomposition times of even the degradable components of bio-plastics will be prolonged, increasing the probability of the plastic being fouled and subsequently reducing UV permeation on which the degradation process relies (Andrady, 2011; Moore, 2008; O'Brine and Thompson, 2010). Once decomposition does finally occur, microplastics will be released into the marine environment (Roy et al., 2011). 


\section{Sources and transfer of microplastics into the marine environment}

Marine litter results from the indiscriminate disposal of waste items that are either directly or indirectly transferred to our seas and oceans (Lozano and Mouat, 2009; Ryan et al., 2009). In this section, we look at several sources of plastic litter and discuss both direct and indirect routes by which plastic can enter the marine environment. Whilst the emphasis of this review is on microplastics, in this section we also consider the indiscriminate disposal of macroplastics, as, with time, they have the potential to degrade into secondary microplastics.

Plastic litter with a terrestrial source contributes $\sim 80 \%$ of the plastics found in marine litter (Andrady, 2011). Such plastics include primary microplastics used in cosmetics and air-blasting, improperly disposed "user" plastics and plastic leachates from refuse sites. With approximately half the world's population residing within fifty miles of the coast, these kinds of plastic have a high potential to enter the marine environment via rivers and wastewater-systems, or by being blown off-shore (Moore, 2008; Thompson, 2006). Microplastics used both in cosmetics and as air-blasting media can enter waterways via domestic or industrial drainage systems (Derraik, 2002); whilst waste-water treatment plants will trap macroplastics and some small plastic debris within oxidation ponds or sewage sludge, a large proportion of microplastics will pass through such filtration systems (Browne et al., 2007; Fendall and Sewell, 2009; Gregory, 1996). Plastics that enter river systems - either directly or within waste-water effluent or in refuse site leachates - will then be transported out to sea. A number of studies have shown how the high unidirectional flow of freshwater systems drives the movement of plastic debris into the oceans (Browne et al., 2010; Moore et al., 2002). Using water samples from two Los Angeles (California, USA) rivers collected in 2004-2005, Moore et al. (2008) quantified the amount of plastic fragments present that were $<5 \mathrm{~mm}$ in diameter. Extrapolating the resultant 
data revealed that these two rivers alone would release over 2 billion plastic particles into the marine environment over a 3-day period. Extreme weather, such as flash flooding or hurricanes, can exacerbate this transfer of terrestrial debris from land to sea (Barnes et al., 2009; Thompson et al., 2005). Work conducted by Moore et al. (2002) showed neustonic litter (small, surface plastic debris) $<4.75 \mathrm{~mm}$ in diameter in Californian waters near the mouth of a modified Los Angeles stormwater conveyance system increased from 10 plastic items $/ \mathrm{m}^{3}$ to 60 plastic items $/ \mathrm{m}^{3}$ following a storm. The work further showed how increased water volume in the river, due to the recent storm, resulted in litter being deposited at even greater distances from the river mouth. Similarly, in a study by Lattin et al. (2004), microplastic concentrations $0.8 \mathrm{~km}$ off the southern Californian coast jumped from an average $<1 \mathrm{item} / \mathrm{m}^{3}$, to $18 \mathrm{items} / \mathrm{m}^{3}$ following a storm.

Coastal tourism, recreational and commercial fishing, marine vessels and marineindustries (e.g. aquaculture, oil-rigs) are all sources of plastic that can directly enter the marine environment, posing a risk to biota both as macroplastics, and as secondary microplastics following long-term degradation. Tourism and recreational activities account for an array of plastics being discarded along beaches and coastal resorts (Derraik, 2002), although it is worth noting that marine debris observed on beaches will also arise from beaching of materials carried on in-shore- and ocean currents (Thompson, 2006). Fishing gear is one of the most commonly noted plastic debris items with a marine source (Andrady, 2011). Discarded or lost fishing gear, including plastic monofilament line and nylon netting, is typically neutrally buoyant and can therefore drift at variable depths within the oceans. This is particularly problematic due to its inherent capacity for causing entanglement of marine biota, known as "ghost fishing" (Lozano and Mouat, 2009). Historically, marine vessels have been a significant contributor to marine litter, with estimates indicating that during the 1970s the global commercial fishing fleet dumped over 23,000 tons of 
plastic packaging materials (Pruter, 1987). In 1988, an international agreement (MARPOL 73/78 Annex V) was implemented banning marine vessels from disposing of plastic waste at sea; however, it is widely considered that a lack of enforcement and education has resulted in shipping remaining a dominant source of plastic in the marine environment (Derraik, 2002; Lozano and Mouat, 2009), contributing an estimated 6.5 million tons of plastic to the oceans in the early 1990s (Derraik, 2002).

Another notable source of plastic debris stems from the manufacture of plastic products that use granules and small resin pellets, known as 'nibs', as their raw material (Ivar do Sul et al., 2009; Mato et al., 2001; Pruter, 1987). In the US alone, production rose from 2.9 million pellets in 1960 to 21.7 million pellets by 1987 (Pruter, 1987). Through accidental spillage during transport, both on land and at sea, inappropriate use as packing materials and direct outflow from processing plants, these raw materials can enter aquatic ecosystems. In an assessment of Swedish waters using an $80 \mu \mathrm{m}$ mesh, KIMO Sweden found typical microplastic concentrations of $150-2,400$ microplastics $/ \mathrm{m}^{3}$, but in a harbour adjacent to a plastic production facility, the concentration was $102,000 / \mathrm{m}^{3}$ (Lozano and Mouat, 2009). However, resin pellets are by no means localised: they have been identified in marine systems worldwide, including mid-ocean islands with no local plastic production facilities (Ivar do Sul et al., 2009; Pruter, 1987). Concentrations of these pellets can also be highly variable: studies conducted in the 1970s and 1980s revealed pellet concentrations of $18 / \mathrm{km}^{2}$ off the New Zealand coast, but $3,500 / \mathrm{km}^{2}$ in the Sargasso Sea (Pruter, 1987). In 1991, under the auspices of the US Environmental Protection Agency, many American plastic manufacturers voluntarily committed to preventing or recapturing spilled pellets, an agreement that may explain significant decreases in quantities of resin pellets identified in the North Atlantic between 1986 and 2008 (Law et al., 2010). More recently, Operation Cleansweep (www.opcleansweep.org), a joint initiative of the American Chemistry Council and 
Society of the Plastics Industry, is aiming for industries to commit to zero pellet loss during their operations.

\section{Assessing microplastic abundance}

Within the marine environment, plastic is widely considered the primary constituent of 'marine debris', a category that includes both anthropogenic litter (e.g. glass, metal, wood), and naturally occurring flotsam (e.g. vegetation, pumice) (Barnes et al., 2009; Moore, 2008; Ryan et al., 2009; Thompson et al., 2004). However, small plastic debris ( $<0.5 \mathrm{~mm}$ in diameter) is considered a widely under-researched component of marine debris (Doyle et al., 2011) due to the difficulties in assessing the abundance, density and distribution of this contaminant within the marine environment. Quantifying the input of plastics into the marine environment is precluded by the array of pathways by which plastics may enter the oceans and would require accurate timescales of the length at which plastics remain at sea prior to degradation (Ryan et al., 2009). Meanwhile, quantifying debris that has already reached the marine environment is complicated by the vastness of the oceans compared to the size of the plastics being assessed. Spatial and temporal variability owing to oceanic currents and seasonal patterns further complicate this issue (Doyle et al., 2011; Ryan et al., 2009). Nevertheless, a suite of sampling techniques has been developed that allow the presence of small plastic debris to be determined. These include: (1) beach combing; (2) sediment sampling; (3) marine trawls; (4) marine observational surveys; and (5) biological sampling.

Beach combing is considered the easiest of the available techniques to conduct, requiring little logistical planning and relatively low costs (MCS, 2010). Typically carried out by researchers and environmental awareness groups, this technique involves collecting and identifying all litter items, in a systematic approach, along a 
specified stretch of coastline. By repeating the beach combing process on a regular basis, accumulation of plastic debris can be monitored over time (Ryan et al., 2009). This technique is particularly useful for determining the presence of macroplastics and plastic resin pellets, termed 'Mermaid's Tears' by beach combers, but microplastics, especially those too small to be observed by the naked eye, are likely to go unnoticed using such a technique. Furthermore, as plastic debris along a coastline will consist of both litter left by recreational beach users and debris deposited by the sea, it must be considered that beach combing data represents a mix of terrestrial litter and marine debris, and therefore may not provide an accurate indicator of plastic debris in the marine environment itself (OSPAR, 2007).

Sediment sampling allows benthic material from beaches, estuaries and the seafloor to be assessed for the presence of microplastics (Claessens et al., 2011). To separate any plastics from the benthic material, saline water or mineral salts can be added to the sediment samples to increase water density, permitting lower-density microplastics to be separated via flotation. Visible, denser plastic fragments can be removed by hand under a microscope (Andrady, 2011; Thompson et al., 2004). A lipophilic dye (e.g. Nile Red) can then be used to stain the plastics to assist identification using a range of microscopy techniques (Andrady, 2011). Using Fourier-Transform Infrared Spectroscopy (FT-IR), items of interest can then be confirmed as plastic by comparing spectra of the samples with that of known polymers (Barnes et al., 2009; Thompson et al., 2004).

Microplastics within the water column can be collected by conducting a trawl along a transect (i.e. manta trawls for sampling surface water, bongo nets for collecting midwater levels and benthic trawls to assess the seabed) using fine meshes (Browne et al., 2010; Ryan et al., 2009; Thompson et al., 2004). The presence of microplastics can then be determined by examining the samples under a microscope, or allowing 
evaporation of the seawater and investigating the residue left behind (Andrady, 2011). Despite the heterogeneous nature of plastics within the ocean, sufficient transects and repeats allow for both spatial and temporal patterns in plastic abundance to be determined in a variety of marine ecosystems (Ryan et al., 2009). Typically, $330 \mu \mathrm{m}$ aperture meshes have been used for many of the microplastic trawls documented in this review, but it is important to note that using meshes with different apertures can produce large variations in the quantity of microplastics collected: by utilising $80 \mu \mathrm{m}$ meshes, KIMO Sweden found microplastics at 100,000 times higher concentrations than when using $450 \mu \mathrm{m}$ meshes (Lozano and Mouat, 2009). In contrast, an Algalita Marine Research Foundation survey of the North Pacific central gyre, conducted in 1999, identified 9,470 plastic fragments with a 1 $\mathrm{mm}$ mesh, but decreasingly smaller quantities of finer sized particles when using smaller-aperture meshes $(4,646$ microplastics with a $0.5 \mathrm{~mm}$ mesh, and just 2,626 microplastics using a $0.3 \mathrm{~mm}$ mesh) (Moore, 2008). Long-term data from Continuous Plankton Recorders (CPRs) are of particular benefit to determining microplastic abundance in the open ocean. These are specialised units designed to constantly sample plankton within $280 \mu \mathrm{m}$ silkscreen-meshes, whilst being towed behind vessels along fixed routes (Thompson et al., 2004). Archived CPR samples, held by the Sir Alastair Hardy Foundation for Ocean Science (SAHFOS) have helped evaluate the prevalence of microplastics in the Northwest Atlantic throughout the past fifty years. The importance of CPR data in assessing microplastic abundance has led SAHFOS to include the presence of microplastics in their analysis of all future samples (Richardson et al., 2006; Thompson et al., 2004).

Marine observational surveys allow divers or observers on boats and in submersibles to record the size, type and location of visible plastic debris. While this technique is effective at detecting macroplastics over relatively large areas, microplastics will often go undetected, and - as debris is not collected - the litter can undergo no 
further assessment (Pruter, 1987; Ryan et al., 2009). Furthermore, the subjective nature of observational work leaves such censuses open to bias (Ryan et al., 2009).

Finally, biological sampling involves examining plastic fragments consumed by marine biota. A number of marine organisms can mistake plastic debris for prey (Blight and Burger, 1997; Tourinho et al., 2010; van Franeker et al., 2011). By dissecting beached marine animals, or by instigating regurgitation in some seabirds, their gut contents can be analysed for the presence of plastics, which can then be identified and quantified (van Franeker, 2010). The Fulmar has routinely been used to assess the abundance of plastic debris at sea for some time and the abundance of microplastics within the stomachs of Fulmars has now become one of the ecological quality assessment markers used by OSPAR to assess the abundance of plastic debris at sea (van Franeker et al., 2011). Whilst migration and movement of this ocean foraging seabird precludes matching their plastic load with specific locales, regional differences and trends over time have become apparent (Blight and Burger, 1997; Tourinho et al., 2010; van Franeker, 2010).

\section{Spatial and temporal trends of microplastics in the marine environment}

Plastic litter has permeated marine ecosystems across the globe (Derraik, 2002; Lozano and Mouat, 2009; Ryan et al., 2009). Driven by ocean currents, winds, river outflow and drift (Barnes et al., 2009; Martinez et al., 2009; Ng and Obbard, 2006) plastic debris can be transported vast distances to remote, otherwise pristine, locations, including mid-ocean islands (Ivar do Sul et al., 2009), the poles (Barnes et al., 2010) and the ocean depths (Lozano and Mouat, 2009). However, whilst plastic litter may be found throughout the marine environment, the distribution of this debris is heterogeneous (Martinez et al., 2009; Moore, 2008). In this section we discuss how microplastics accumulate along coastlines and within mid-ocean gyres, examine 
the variable position of microplastics within the water column and consider microplastic abundance over time.

\section{Accumulation of microplastics}

Coastlines receive plastic litter from both terrestrial and marine sources; terrestrial sources of litter will typically dominate close to urban areas, sites of tourism and near river outflows, whilst marine debris will be deposited along shorelines when caught in near-shore currents (Ryan et al., 2009). Using sediment analysis, Thompson et al. (2004) have found microplastics, consisting of nine different polymers, in 23 of 30 estuarine, beach and sub-tidal sediment samples taken around Plymouth, UK, including microscopic fibres and fragments typically derived from clothing, packaging and rope. Further work showed that microplastics were present in beach sediments throughout the UK. Browne et al. (2010) used the same methodology to quantify microplastics in sediment throughout the Tamar estuary (Plymouth, UK), identifying 952 items in 30 sediment samples. An abundance of microplastics have also been found in productive coastal ecosystems off Alaska and California, where nutrient upwelling results in high densities of planktonic organisms (Doyle et al., 2011). Using $505 \mu \mathrm{m}$ meshes during surface plankton trawls for the National Oceanic and Atmospheric Administration (NOAA), Doyle et al (2011) found an abundance of plastic fragments derived from the breakdown of larger plastic debris, in addition to plastic fibres and pellets, although concentrations were significantly lower than those found in the adjacent North Pacific gyre. The source of this plastic debris was unable to be verified; however, it was suggested that the high concentration of plastics in southern Californian waters during winter was linked to urban run-off from major conurbations, whilst a marine source was more likely during the summer months when currents altered. After conducting beach surveys throughout the remote midAtlantic archipelago of Fernando de Noronha, Ivar do Sul et al (2009) identified 
plastic pre-production resin pellets on the windward beaches of the archipelago - yet no plastic-production facilities exist in the region. Therefore, it was hypothesised that they were brought to the remote location via trans-oceanic currents before being trapped in in-shore currents and washed ashore. Similarly, a survey of beaches on the island of Malta, in the Mediterranean Sea, found an abundance of disc- and cylindrical-shaped plastic resin pellets $(1.9-5.6 \mathrm{~mm}$ in diameter) on all beaches surveyed (Turner and Holmes, 2011). The highest concentrations of pellets, in some cases in excess of 1,000 pellets $/ \mathrm{m}^{2}$, were found along the high-tide mark; the majority of the pellets were yellow or brown in colour, caused by photo-oxidative damage indicative of their longevity within the marine environment. The presence of so many plastics on a shoreline can dramatically alter the physio-chemical properties of the beach sediment. In a recent study, vertical sediment cores were taken from beaches in Hawaii and analysed (Carson et al., 2011). The presence of plastic debris not only increased the permeability of the sediment, but also decreased its heat absorbance so that the sediment would reach lower maximal temperatures than sediment without plastics present. Such differences could affect marine biota; for example, lower maximal temperatures might affect sex-determination in turtle eggs, and greater permeability will increase the probability of desiccation in sedimentdwelling organisms.

Oceanographic modelling indicates a large proportion of floating debris reaching the ocean will accumulate in gyres - the centre of vast anti-cyclonic, sub-tropical ocean currents. Using satellite-tracked "drifters" placed throughout the South Pacific ocean, Martinez et al (2009) mapped the average trajectories of ocean currents, drift and eddies over time; the team found that, whilst some trackers were caught in nearshore currents, the majority fed into the south Pacific gyre from where they could not easily escape (Law et al., 2010; Martinez et al., 2009). Lagrangian drifters have also been used in a more recent study, indicating a high proportion of floating marine 
debris will end up in ocean gyres (Maximenko et al., 2011). Data accumulated from over 6,000 plankton tows conducted between 1986 and 2008 in the North Atlantic Ocean and Caribbean Sea, found plastic in $60 \%$ of the samples (Law et al., 2010). Mapping the plastic concentrations of each transect, Law et al. (2010) revealed distinct spatial patterns of plastic in these areas, with highest concentrations (83\% of total plastic sampled) found in sub-tropical latitudes. The highest concentration was mapped to the North Atlantic gyre, with $20,328( \pm 2,324)$ pieces $/ \mathrm{km}^{2}$. Due to the concentrations of plastic found it was impossible to determine the sources of such debris, but use of trackers suggested much of the eastern seaboard of the US fed into the gyre, taking debris 60 days on average to reach the gyre sited over $1,000 \mathrm{~km}$ away. Even higher plastic concentrations have been recorded in the North Pacific gyre: conducting 11 transects using a $333 \mu \mathrm{m}$ manta-trawl, Moore et al. (2001) identified plastics in the majority of their tows, with an average density of 334,271 plastic fragments $/ \mathrm{km}^{2}$. Such work has led to significant media attention, with the North Pacific gyre being described "plastic soup" and coined as the "great Pacific garbage patch" (Kaiser, 2010).

\section{Microplastics in the water column}

Plastics consist of many different polymers and, depending on their composition, density and shape, can be buoyant, neutrally-buoyant or sink. As such, microplastics may be found throughout the water column. Low-density microplastics are predominantly found in the sea-surface microlayer, as documented by numerous studies presenting data from surface trawls (Derraik, 2002; Gregory, 1996). However, there is evidence that their position in the water column can vary: in estuarine habitats, low-density plastics, such as polypropylene and polyethylene, will be submerged if they meet water fronts. Furthermore, there is growing evidence that the attachment of fouling organisms can cause buoyant microplastics to sink (Barnes et 
al., 2009; Browne et al., 2010; Derraik, 2002; Thompson et al., 2004). Plastic debris in the marine environment can rapidly accumulate microbial biofilms, which further permit the colonisation of algae and invertebrates on the plastics' surface, thus increasing the density of the particle (Andrady, 2011). The speed at which biofouling may occur was recently demonstrated using polyethylene plastic bags submerged in seawater $\left(16.2{ }^{\circ} \mathrm{C}\right)$ in Plymouth harbour (UK); a biofilm was visible after just one week, and analysis showed a significant increase in microbial density over the 3week experiment (Lobelle and Cunliffe, 2011). Notably, the plastic became less buoyant over time, and by the end of the experiment the plastic moved away from the surface and appeared neutrally buoyant. When assessing plastic litter in the North Pacific gyre, Moore et al. (2001) randomly sampled debris for signs of fouling organisms. Only a small proportion (8.5\%) of surface debris was colonised, and fouling decreased with particle size. However, at a depth of $10 \mathrm{~m}$, a higher proportion of plastics debris was fouled with algae and diatoms. More recently, an analysis of microplastics $(<1 \mathrm{~mm})$ collected in surface tows from the western North Atlantic Ocean between 1991 and 2007, has shown evidence of fouling (Morét-Ferguson et al., 2010). The study found low-density polymers (e.g. polypropylene and polyethylene) with higher densities than the same polymer found on beaches, concluding the increase in density resulted from biofouling at sea. Despite increases of plastic debris entering the marine environment throughout the last century, Law et al. (2010) found no significant change in microplastic abundance in the Northwest Atlantic over the past twenty years. To test whether new input of microplastics was compensated for by sedimentation of biofouled plastics to greater depths, they analysed material from sediment traps deployed at 500 to $3,200 \mathrm{~m}$ depths close to the north Atlantic gyre, but found no significant accumulation of plastic particles. The fate of fouled microplastics in gyres has now become a key research area for the 5 Gyres Project, in association with the Algalita Marine Research Foundation (AMRF) (Eriksen and Cummins, 2010). 
High-density microplastics, including polyvinylchloride, polyester and polyamide, are likely found in their largest quantities in the benthos. However, determining the magnitude of microplastic debris on the seafloor is hindered by cost and difficulties of sampling (Barnes et al., 2009). While 'Fishing for Litter' schemes, conducted in the Netherlands and Scotland, and submersible video-recordings can document the quantity of macroplastics present on the seafloor (Lozano and Mouat, 2009; Watters et al., 2010), microplastics will fall below the lower limits of detection of these sampling methods. Therefore, quantification of microplastics in the benthos relies on sediment-grabs and benthic trawls using fine meshes. A recent study has found some of the highest microplastic concentrations within sediment thus far. Microplastics, $<1 \mathrm{~mm}$ in diameter, consisting of fibres, granules, pellets and films, were found in all beach, harbour and sub-littoral sediment samples taken off the Belgian coast (Claessens et al., 2011); the highest microplastic concentration ( 391 microplastics $/ \mathrm{kg}$ of dry sediment) was found in a harbour sediment sample, probably due to the local anthropogenic activity, river run-off and trapping of sediments. It has been documented that high-density microplastics can be temporarily suspended within the water-column in smaller numbers resulting from turbulence. High-density microplastics can remain in suspension when entering the sea through estuaries due to tidal fronts, high-flow rate or because of a large-surface area (Brown et al. 2010). Only when momentum is lost will these dense polymers inevitably sink (Barnes et al., 2009). Microplastics on the seabed may also be re-suspended resulting from turbulence: Lattin et al. (2004) quantified microplastic concentrations $>333 \mu \mathrm{m}$ at varying depths, $0.8 \mathrm{~km}$ and $4.5 \mathrm{~km}$ off the southern Californian coast. At the off-shore site, microplastics were most abundant close to the seafloor (6 items $/ \mathrm{m}^{3}$ ), but were redistributed throughout the water column after a storm (Lattin et al., 2004). 
Temporal changes in microplastic abundance within the marine environment

Since the 1940s, when the mass production of plastics began in earnest, the volume of plastic produced has risen rapidly. With legislation to curb the indiscriminate disposal of plastic waste emerging slowly, plastic debris entering the marine environment increased in parallel with rates of production during this time (Moore, 2008; Ryan, 2009; Barnes 2009). Continuous fragmentation of larger plastic debris and the rising popularity of "plastic scrubbers" appears to have increased the volume of microplastic debris in the oceans, resulting in a decrease in the average size of plastic litter over time (Barnes et al., 2009). This was highlighted by Thompson et al. (2004), who demonstrated that microplastic concentrations in the 1980s and 1990s were significantly greater than those in the 1960s and 1970s in an analysis of CPR samples from the North Sea and Northwest Atlantic. Furthermore, incidence of plastic ingestion by Fulmars (ocean-foraging seabirds), washed ashore in the Netherlands, increased from $91 \%$ to $98 \%$ between the 1980 s and 2000 , whilst the average consumption doubled from 15 to 30 plastic fragments per bird during this period (van Franeker et al., 2011).

Concentration trends within the past decade are not overtly apparent, and there is some debate as to whether levels of plastic debris are still increasing or have stabilised. The study by Thompson et al. (2004) indicated minimal change in microplastic contamination between the 1980s and 1990s. Similarly, an evaluation of $>6,100$ surface trawls conducted throughout the Northwest Atlantic Ocean found no significant difference in microplastic abundance over a 22 year period (Law et al. 2010). The average number of plastics debris items consumed by Fulmars, beached on the shores of the Netherlands, decreased slightly from the mid-1990s, but has remained relatively stable since the turn of the century, currently averaging 26 plastic fragments per bird (van Franeker et al., 2011). In contrast, Claessens et al. (2011) 
indicate that microplastic concentrations have steadily increased over the past two decades. Analysis of sediment cores taken along the Belgian coast indicates microplastic pollution tripled from $\sim 55$ microplastics/kg of dry sediment (1993-2000) to 156 microplastics/kg of dry sediment (2005-2008), in line with global production rates. However, use of sediment cores is a new technique, and bio-turbation from tourism or sediment-dwelling biota might have affected this data.

Any further conclusions are hampered by both a lack of studies that have specifically considered trends of microplastic abundance over time. Meta-studies are difficult to develop due to varieties of sampling methodologies, huge spatial variations in microplastic abundance, and lack of standardised size definitions of microplastics (Ryan, 2009; Barnes 2009).

\section{Impact of microplastics on the marine environment}

Whilst it is apparent that microplastics have become both widespread and ubiquitous, information on the biological impact of this pollutant on organisms in the marine environment is only just emerging (Barnes et al., 2009; Gregory, 1996; Ryan et al., 2009). The possibility that microplastics pose a threat to biota, as their small size makes them available to a wide range of marine organisms, is of increasing scientific concern (Barnes et al., 2009; Derraik, 2002; Fendall and Sewell, 2009; Lozano and Mouat, 2009; $\mathrm{Ng}$ and Obbard, 2006; Thompson et al., 2004). In addition to potential adverse effects from ingesting the microplastics themselves, toxic responses could also result from (a) inherent contaminants leaching from the microplastics, and (b) extraneous pollutants, adhered to the microplastics, disassociating. 
Microplastic ingestion

Owing to their small size and presence in both pelagic and benthic ecosystems, microplastics have the potential to be ingested by an array of marine biota (Betts, 2008; Thompson et al., 2009a). Observing microplastic ingestion in the wild is methodologically challenging (Browne et al., 2008), but an increasing number of studies are reporting microplastic ingestion throughout the food-chain.

Table 1 lists a number of laboratory experiments demonstrating that marine organisms, including zooplankton, invertebrates and echinoderm larvae, ingest microplastics (Bolton and Havenhand, 1998; Brillant and MacDonald, 2002; Hart, 1991; Wilson, 1973). Furthermore, phagocytic uptake of nanoplastics in a heterotrophic ciliate has been demonstrated using fluorescent nanospheres (Pace and Bailiff, 1987). These lower-trophic level organisms are particularly susceptible to ingesting microplastics as many of them are indiscriminate feeders with limited ability to differentiate between plastic particles and food (Moore, 2008). A study investigating the colour and size distribution of microplastics in the North Pacific Ocean hypothesised that planktonic organisms will most commonly mistake white and lightly-coloured plastic fragments for prey (Shaw and Day, 1994). As low-density "user" plastics (e.g. polyethylene and polystyrene) are buoyant, microplastics are abundant near the sea surface. Therefore, microplastics will be widely available to a host of planktonic organisms, including the larval stages of a variety of commercially important species, that reside within the euphotic zone (Fendall and Sewell, 2009; Gregory, 1996). This contact between plankton and microplastics is hypothetically exacerbated in gyres, as plankton populations are low whilst microplastic concentrations are high, resulting from plastic accumulation by ocean currents (Moore, 2008). 
A range of marine biota, including seabirds, crustaceans and fish, can ingest microplastics (Blight and Burger, 1997; Tourinho et al., 2010). Plastic fragments were first identified in the guts of sea birds in the 1960s, when global plastic production was less than 25 million tonnes per annum (Ryan et al., 2009; Thompson et al., 2009b). In 1982, a team in the Netherlands found $94 \%$ of fulmars sampled contained plastics, with an average of 34 plastic fragments per individual. Since, incidence and number of fragments consumed has remained high, although the mass of plastic found in each bird has decreased significantly in recent years (Lozano and Mouat, 2009; van Franeker, 2010). Dissection of planktivorous mesopelagic fish, caught in the North Pacific central gyre, revealed microplastics in the guts of $\sim 35 \%$ of the fish sampled (Boerger et al., 2010). Plastc fibres, fragments and films were also found in the stomachs of 13 of 141 mesopelagic fish caught in the North Pacific gyre (Davison and Asch, 2011). In the Clyde Sea (Scotland), 83\% of Nephrops sp. collected had ingested plastics. This commercially important, omnivorous, benthic-dwelling crustacean mainly ate sections of monofilament line and fragments of plastic bags (Murray and Cowie, 2011). Plastic fibres found in the environment can be as small as $1 \mu \mathrm{m}$ in diameter, and $15 \mu \mathrm{m}$ in length, making them available to minute planktonic species (Frias et al., 2010). Such fibres may be particularly hazardous as they may clump and knot, potentially preventing egestion (Murray and Cowie, 2011). In all these examples, these animals might have ingested microplastics voluntarily, which they confuse for their prey. Alternatively, microplastic ingestion may result from eating lower trophic organisms that have themselves consumed microplastics (Browne et al., 2008; Fendall and Sewell, 2009). This process was recently demonstrated by providing small fish, which had previously eaten plastic fibres, to Nephrops sp.; after a 24-hour exposure period, all the Nephrops sp. had plastic fibres in their guts from eating the fish (Murray and Cowie, 2011). 
It is yet to be established whether the ingestion of non-polluted microplastics have any significant adverse health effects on biota (e.g. morbidity, mortality or reproductive success) (Zarfl et al., 2011). Microplastics may present a mechanical hazard to small animals once ingested, similar to the effects observed for macroplastics and larger animals (Barnes et al., 2009; Fendall and Sewell, 2009): plastic fragments might block feeding appendages or hinder the passage of food through the intestinal tract (Tourinho et al., 2010) or cause pseudo-satiation resulting in reduced food intake (Derraik, 2002; Thompson, 2006). However, Thompson (2006) and Andrady (2011) note that numerous marine organisms have the ability to remove unwanted materials (e.g. sediment, natural detritus and particulates) from their body without causing harm, as demonstrated using polychaete worms, which ingested microplastics from their surrounding sediment, then egested them in their faecal casts (Thompson et al., 2004). Nevertheless, once ingested, there is the potential for microplastics to be absorbed into the body upon passage through the digestive system via translocation. Translocation of polystyrene microspheres was first shown in rodents and humans, and has also been demonstrated for mussels using histological techniques and fluorescence microscopy (Browne et al., 2008). Mytilus edulis were able to ingest $2 \mu \mathrm{m}$ and $4 \mu \mathrm{m}$ microplastics via the inhalant siphon, which the gill filtered out and transported to the labial palps for digestion or rejection. Translocation was proven following the identification of $3 \mu \mathrm{m}$ and $9.6 \mu \mathrm{m}$ fluorescently tagged microspheres in the mussels' haemolymph (circulatory fluid), 3 days after exposure. Microspheres were present in the circulatory system for up to 48 days after exposure, although there was no apparent sub-lethal impact (measured as oxidative status and phagocytic ability of the haemocytes) (Browne et al., 2008). However, Köhler (2010) describes a pronounced immune response and granuloma formation in the digestive glands of blue mussels exposed to microplastics. 
Microplastics and plasticiser leachates

Although plastics are typically considered as biochemically inert (Roy et al., 2011; Teuten et al., 2009), plastic additives, often termed "plasticisers", may be incorporated into plastics during manufacture to change their properties or extend the life of the plastic by providing resistance to heat (e.g. polybrominateddiphenyl ethers), oxidative damage (e.g. nonylphenol) and microbial degradation (e.g. triclosan) (Browne et al., 2007; Thompson et al., 2009b). These additives are an environmental concern since they both extend the degradation times of plastic and may, in addition, leach out, introducing potentially hazardous chemicals to biota (Barnes et al., 2009; Lithner et al., 2011; Talsness et al., 2009).

Incomplete polymerisation during the formation of plastics allows additives to migrate away from the synthetic matrix of plastic; the degree to which these additives leach from plastics is dependent on the pore size of the polymer matrix, which varies by polymer, the size and properties of the additive and environmental conditions (e.g. weathering) (Moore, 2008; $\mathrm{Ng}$ and Obbard, 2006; Teuten et al., 2009). For example, phthalates are emollients that soften plastics by reducing the affinity between molecular chains within the synthetic polymer matrix (Oehlmann et al., 2009; Talsness et al., 2009). In PVC, phthalates can constitute up to $50 \%$ of the plastic's weight (Oehlmann et al., 2009). Meanwhile, Bisphenol A is a constituent monomer in polycarbonate which is widely used in food and beverage containers. Neither compound is persistent, but their instability within plastic products facilitates leaching and their high prevalence in aquatic environments has been widely reported, particularly in landfill leachates (vom Saal and Myers, 2008).

Due to the large surface-area-to-volume ratio of microplastics, marine biota may be directly exposed to leached additives after microplastics are ingested. Such additives 
and monomers may interfere with biologically important processes, potentially resulting in endocrine disruption, which in turn can impact upon mobility, reproduction and development, and carcinogenesis (Barnes et al., 2009; Lithner et al., 2009; Lithner et al., 2011). Commonly used additives, including polybrominated diphenyl ethers, phthalates and the constituent monomer bisphenol A, are renowned for being endocrine-disrupting chemicals as they can mimic, compete with or disrupt the synthesis of endogenous hormones (Talsness et al., 2009). Hormonal imbalance can cause permanent morphological issues in organisms in developmental stages, or sexual disruption in adults. Phthalates have been associated with a range of molecular and whole-organism effects in aquatic invertebrates and fish, including genotoxic damage (micronuclei and apoptosis in mussel haemocytes), inhibited locomotion in invertebrates and intersex conditions in fish (Oehlmann et al., 2009). Bisphenol $A$ is both an oestrogen agonist and an androgen antagonist that can differentially affect reproduction and development depending on its concentration and the organism affected; at concentrations in the region of $\mu \mathrm{g} / \mathrm{l}$, Bisphenol $\mathrm{A}$ can be acutely toxic to both crustaceans and insects. Chronic and widespread exposure of human populations to Bisphenol $\mathrm{A}$ has further been associated with chronic health effects, including heart disease, diabetes and alterations in circulating hormone levels (Galloway et al., 2010; Lang et al., 2008). Although it has been shown that plasticisers can induce negative biological effects within the $\mathrm{ng} / \mathrm{l}-\mu \mathrm{g} / \mathrm{l}$ range, Oehlmann et al. (2009) note there has been relatively little research into the chronic effects of these additives in long-term exposures to aquatic species.

\section{Microplastics and adhered pollutants}

Marine plastic debris, in particular microplastics with their large surface area to volume ratio, are susceptible to contamination by a number of waterborne-pollutants, including aqueous metals (Betts, 2008; Ashton et al., 2010), endocrine disrupting 
chemicals ( $\mathrm{Ng}$ and Obbard, 2006) and persistent organic pollutants (POPs), also referred to as hydrophobic organic contaminants (HOCs) (Rios et al., 2007).

Such chemicals are typically found at their highest concentrations in the sea-surface microlayer, where low-density microplastics are most abundant as well $(\mathrm{Ng}$ and Obbard, 2006; Rios et al., 2007; Teuten et al., 2009). POPs, which include polychlorinated biphenyls (PCBs), PAHs and organochlorine pesticides (e.g. DDT, DDE), are stable, lipophillic chemicals that will adhere and concentrate on the hydrophobic surface of plastics, with environmental concentrations recorded in the ng/g - $\mu \mathrm{g} / \mathrm{g}$ range (Teuten et al., 2007; Teuten et al., 2009; Barnes et al., 2009). Using equilibrium partitioning modelling, the adsorption coefficients $(\mathrm{Kd})$ of the priority pollutant phenanthrene were calculated for a range of plastic polymers in seawater and natural sediments (Teuten et al., 2007). Phenanthrene readily sorbs to small plastics, preferentially adhering to polyethylene, likely due to larger molecular cavities in this polymer. In environmentally relevant conditions, phenanthrene was more likely to adhere to plastics than to sediment. However, if heavily polluted microplastics come into contact with non-contaminated sediments, the concentration gradient would permit desorption of phenanthrene to organic matter in the sediment.

Evidence of microplastic contamination has been highlighted by several studies conducted in recent years. Mato et al. (2001) identified PCBs, nonylphenol and DDE on polypropylene resin pellets collected from Japanese waters at similar or higher concentrations than those found in sediments. In a further experiment, virgin resin pellets were shown to adsorb contaminants from seawater within a 6-day exposure period. Although adsorption was constant, maximal concentrations were not reached in this time, indicating adsorption is not a rapid process. Rios et al. (2007) used GCMS to detect sorbed contaminants on plastic pellets in Japanese waters; 4,4-DDE was found on all samples, up to a concentration of $5,600 \mathrm{ng} / \mathrm{g}$, and PCBs were 
observed on all but four samples with concentrations of 39-1,200 ng/g. Teuten et al. (2007) observed PCBs at concentrations $10^{6}$ higher on polystyrene pellets than in surrounding water. Microplastics found on two Portuguese beaches contained PAH concentrations ranging from $0.2-319.2 \mathrm{ng} / \mathrm{g}$, and PCBs from $0.02-15.56 \mathrm{ng} / \mathrm{g}$ (Frias et al., 2010). Analysis of plastic fragments $(<10 \mathrm{~mm})$ sampled from pelagic and neritic stations, revealed a range of pollutants including PCBs, PAHs, DDTs and its metabolites, PBDEs and bisphenol A were adhered to the plastics' surface at concentrations of $1-10,000 \mathrm{ng} / \mathrm{g}$ (Hirai et al., 2011).

Microplastic debris coated with POPs may be transported across oceans polluting otherwise pristine ecosystems (Zarfl and Matthies, 2010), or be ingested by marine organisms, thus transferring toxins from the environment to biota (i.e. a "Trojan horse" effect) (Gregory, 1996; Thompson et al., 2005; Thompson et al., 2004). Many POPs are considered toxic, inducing endocrine disruption, mutagenesis and/or carcinogenesis, and may biomagnify in higher-trophic organisms. However, until recent years it was unclear whether contaminants adhered to plastic detritus would disassociate once ingested (Thompson et al., 2004). To determine whether pollutants adhered to microplastics could desorb and cause harm to biota, Teuten et al. (2007) used a partitioning model to assess the disassociation of phenanthrene on microplastics. The model indicated that contaminated microplastics ingested by Arenicola marina, a sediment-dwelling polychaete worm, will sequester a proportion of the sorbed contaminants to the organism. However, if inhabiting clean, organicrich sediment, much of the contaminant was predicted to adhere to the sediment rather than be taken up by the polychaete itself (Teuten et al., 2007; Teuten et al., 2009). Transfer of contaminants from plastic to biota has since been demonstrated. Streaked shearwater chicks were fed with a diet of fish and resin pellets, or fish alone (Betts, 2008; Teuten et al., 2009). Both pellets and fish were obtained from Tokyo Bay and were contaminated with polychlorinated biphenyls (PCBs), at concentrations 
of $51-562 \mathrm{ng} / \mathrm{g}$ for the plastics, and $0.3-0.7 \mathrm{ng} / \mathrm{g}$ for fish. Analysis of preen gland oil, taken every week for 42 days, showed that PCB concentrations increased in both groups of chicks. To determine the uptake of PCBs from the resin pellets alone, lower chlorinated congener PCBs, which were abundant in the resin pellets but in low concentrations in fish, were analysed. Chicks eating plastic pellets showed a significant increase in low congener PCBs, whilst those eating fish alone showed no change.

\section{Conclusions and recommendations for future work}

Over the past decade, increased scientific interest has produced an expanding knowledge base for microplastics. Nevertheless, fundamental questions and issues remain unresolved. An evolving suite of sampling techniques has revealed that microplastics are a ubiquitous and widespread marine contaminant, present throughout the water column. However, disparity in the size definitions of microplastics and lack of comparability of microplastic sampling methodologies hinder our ability to cross-examine quantitative studies to better determine spatial and temporal patterns of this contaminant. The highest abundance of microplastics is typically associated with coastlines and mid-ocean gyres, but the fate of these microplastics is elusive. It is hypothesised that microplastics sink following biofouling, fragment into smaller and smaller polymer fragments and/or are ingested by marine biota. Fully testing such hypotheses is impeded by the complexity of sampling the ocean depths and the difficulty of routinely sampling and detecting smaller-sized fractions of microplastics (including nanoplastics). Laboratory and field-studies have shown the consumption of microplastics in a range of marine biota, although it remains unclear whether microplastic ingestion alone will result in adverse health effects (e.g. mortality, morbidity and reproductive success) or whether such a contaminant can routinely be passed up the food chain. The transfer of toxic 
chemicals to biota via microplastic ingestion is a significant concern. However, few existing studies have conducted toxicity-studies using microplastic vectors. Looking to the future, here we present a list of knowledge gaps we believe deserve further attention from the scientific community (Table 2).

\section{Acknowledgements}

MC is supported by a NERC PhD studentship. This work was supported by grant ME5413 from the Department of the Environment, Fisheries and Rural Affairs, UK.

\section{References}

Andrady, A.L., 2011. Microplastics in the marine environment. Marine Pollution Bulletin 62, 1596-1605.

Andrady, A.L., Neal, M.A., 2009. Applications and societal benefits of plastics, pp. 1977-1984.

Ashton, K., Holmes, L., Turner, A., 2010. Association of metals with plastic production pellets in the marine environment. Marine Pollution Bulletin 60, 20502055.

Barnes, D.K.A., 2002. Biodiversity: Invasions by marine life on plastic debris. Nature 416, 808-809.

Barnes, D.K.A., Galgani, F., Thompson, R.C., Barlaz, M., 2009. Accumulation and fragmentation of plastic debris in global environments. Philosophical Transactions of the Royal Society B: Biological Sciences 364, 1985-1998.

Barnes, D.K.A., Walters, A., Gonçalves, L., 2010. Macroplastics at sea around Antarctica. Marine Environmental Research 70, 250-252. 
Betts, K., 2008. Why small plastic particles may pose a big problem in the oceans. Environmental Science \& Technology 42, 8995-8995.

Blight, L.K., Burger, A.E., 1997. Occurrence of plastic particles in seabirds from the eastern North Pacific. Marine Pollution Bulletin 34, 323-325.

Boerger, C.M., Lattin, G.L., Moore, S.L., Moore, C.J., 2010. Plastic ingestion by planktivorous fishes in the North Pacific Central Gyre. Marine Pollution Bulletin 60, 2275-2278.

Bolton, T.F., Havenhand, J.N., 1998. Physiological versus viscosity-induced effects of an acute reduction in water temperature on microsphere ingestion by trochophore larvae of the serpulid polychaete Galeolaria caespitosa, pp. 2153-2164.

Brillant, MacDonald, 2002. Postingestive selection in the sea scallop (Placopecten magellanicus) on the basis of chemical properties of particles. Marine Biology 141, 457-465.

Browne, M.A., Dissanayake, A., Galloway, T.S., Lowe, D.M., Thompson, R.C., 2008. Ingested Microscopic Plastic Translocates to the Circulatory System of the Mussel, Mytilus edulis (L.). Environmental Science \& Technology 42, 5026-5031.

Browne, M.A., Galloway, T., Thompson, R., 2007. Microplastic - an emerging contaminant of potential concern? Integrated Environmental Assessment and Management 3, 559-561.

Browne, M.A., Galloway, T.S., Thompson, R.C., 2010. Spatial Patterns of Plastic Debris along Estuarine Shorelines. Environmental Science \& Technology 44, 34043409.

Carpenter, E.J., Smith, K.L., 1972. Plastics on the Sargasso Sea Surface, pp. 12401241. 
Carson, H.S., Colbert, S.L., Kaylor, M.J., McDermid, K.J., 2011. Small plastic debris changes water movement and heat transfer through beach sediments. Marine Pollution Bulletin 62, 1708-1713.

Claessens, M., Meester, S.D., Landuyt, L.V., Clerck, K.D., Janssen, C.R., 2011. Occurrence and distribution of microplastics in marine sediments along the Belgian coast. Marine Pollution Bulletin In Press.

Costa, M., Ivar do Sul, J., Silva-Cavalcanti, J., Araújo, M., Spengler, Â., Tourinho, P., 2010. On the importance of size of plastic fragments and pellets on the strandline: a snapshot of a Brazilian beach. Environmental Monitoring and Assessment 168, 299304.

Davison, P., Asch, R.G., 2011. Plastic ingestion by mesopelagic fishes in the North Pacific Subtropical Gyre, pp. 173-180.

Derraik, J.G.B., 2002. The pollution of the marine environment by plastic debris: a review. Marine Pollution Bulletin 44, 842-852.

Doyle, M.J., Watson, W., Bowlin, N.M., Sheavly, S.B., 2011. Plastic particles in coastal pelagic ecosystems of the Northeast Pacific ocean. Marine Environmental Research 71, 41-52.

Eriksen, M., Cummins, A., 2010. Spatial Distribution of Plastic Marine Debris in the Marine Environment, Proceedings from the 2010 AGU Ocean Sciences Meeting (2226 Feb 2010).

Fendall, L.S., Sewell, M.A., 2009. Contributing to marine pollution by washing your face: Microplastics in facial cleansers. Marine Pollution Bulletin 58, 1225-1228.

Frias, J.P.G.L., Sobral, P., Ferreira, A.M., 2010. Organic pollutants in microplastics from two beaches of the Portuguese coast. Marine Pollution Bulletin 60, 1988-1992. 
Galgani, F., Fleet, D., Franeker, J.V., Katsanevakis, S., T.Maes, J.Mouat, Oosterbaan, L., Poitou, I., Hanke, G., Thompson, R., Amato, E., A.Birkun, Janssen, C., 2010. Task Group 10 Report: Marine litter, in: Zampoukas, N. (Ed.), Marine Strategy Framework Directive. JRC, Ifremer \& ICES.

Galloway, T., Cipelli, R., Guralnick, J., Ferrucci, L., Bandinelli, S., Corsi, A.M., Money, C., McCormack, P., Melzer, D., 2010. Daily Bisphenol A Excretion and Associations with Sex Hormone Concentrations: Results from the InCHIANTI Adult Population Study. Environmental Health Perspectives 118, 1603-1608.

Graham, E.R., Thompson, J.T., 2009. Deposit- and suspension-feeding sea cucumbers (Echinodermata) ingest plastic fragments. Journal of Experimental Marine Biology and Ecology 368, 22-29.

Gregory, M.R., 1996. Plastic 'scrubbers' in hand cleansers: a further (and minor) source for marine pollution identified. Marine Pollution Bulletin 32, 867-871.

Gregory, M.R., 2009. Environmental implications of plastic debris in marine settings: entanglement, ingestion, smothering, hangers-on, hitch-hiking and alien invasions. Philosophical Transactions of the Royal Society B: Biological Sciences 364, 20132025.

Hart, M.W., 1991. Particle Captures and the Method of Suspension Feeding by Echinoderm Larvae, pp. 12-27.

Hirai, H., Takada, H., Ogata, Y., Yamashita, R., Mizukawa, K., Saha, M., Kwan, C., Moore, C., Gray, H., Laursen, D., Zettler, E.R., Farrington, J.W., Reddy, C.M., Peacock, E.E., Ward, M.W., 2011. Organic micropollutants in marine plastics debris from the open ocean and remote and urban beaches. Marine Pollution Bulletin 62, 1683-1692. 
Ivar do Sul, J.A., Spengler, A., Costa, M.F., 2009. Here, there and everywhere. Small plastic fragments and pellets on beaches of Fernando de Noronha (Equatorial Western Atlantic). Marine Pollution Bulletin 58, 1236-1238.

Kaiser, J., 2010. The Dirt on Ocean Garbage Patches. Science 328, 1506.

Köhler, A., 2010. Cellular fate of organic compounds in marine invertebrates. Comparative Biochemistry and Physiology - Part A: Molecular \& Integrative Physiology 157, S8-S8.

Lang, I.A., Galloway, T.S., A., S., Henley, W.E., Depledge, M., Bowman, R.B., Melzer, D., 2008. Association of urinary bisphenol A concentration with medical disorders and laboratory abnormalities in adults. Journal of the American Medical Association 300, 1303-1310.

Lattin, G.L., Moore, C.J., Zellers, A.F., Moore, S.L., Weisberg, S.B., 2004. A comparison of neustonic plastic and zooplankton at different depths near the southern California shore. Marine Pollution Bulletin 49, 291-294.

Law, K.L., Moret-Ferguson, S., Maximenko, N.A., Proskurowski, G., Peacock, E.E., Hafner, J., Reddy, C.M., 2010. Plastic Accumulation in the North Atlantic Subtropical Gyre, Science, pp. 1185-1188.

Lithner, D., Damberg, J., Dave, G., Larsson, A., 2009. Leachates from plastic consumer products - Screening for toxicity with Daphnia magna. Chemosphere 74 , $1195-1200$

Lithner, D., Larsson, Å., Dave, G., 2011. Environmental and health hazard ranking and assessment of plastic polymers based on chemical composition. Science of The Total Environment 409, 3309-3324. 
Lobelle, D., Cunliffe, M., 2011. Early microbial biofilm formation on marine plastic debris. Marine Pollution Bulletin 62, 197-200.

Lozano, R.L., Mouat, J., 2009. Marine litter in the North-East Atlantic Region: Assessment and priorities for response. KIMO International.

Martinez, E., Maamaatuaiahutapu, K., Taillandier, V., 2009. Floating marine debris surface drift: Convergence and accumulation toward the South Pacific subtropical gyre. Marine Pollution Bulletin 58, 1347-1355.

Mato, Y., Isobe, T., Takada, H., Kanehiro, H., Ohtake, C., Kaminuma, T., 2001. Plastic Resin Pellets as a Transport Medium for Toxic Chemicals in the Marine Environment. Environmental Science \& Technology 35, 318-324.

Maximenko, N., Hafner, J., Niiler, P., 2011. Pathways of marine debris derived from trajectories of Lagrangian drifters. Marine Pollution Bulletin In Press.

MCS, 2010. Marine Conservation Society - Beachwatch 2009: Methods and full results.

Moore, C.J., 2008. Synthetic polymers in the marine environment: A rapidly increasing, long-term threat. Environmental Research 108, 131-139.

Moore, C.J., Moore, S.L., Weisberg, S.B., Lattin, G.L., Zellers, A.F., 2002. A comparison of neustonic plastic and zooplankton abundance in southern California's coastal waters. Marine Pollution Bulletin 44, 1035-1038.

Morét-Ferguson, S., Law, K.L., Proskurowski, G., Murphy, E.K., Peacock, E.E., Reddy, C.M., 2010. The size, mass, and composition of plastic debris in the western North Atlantic Ocean. Marine Pollution Bulletin 60, 1873-1878. 
Murray, F., Cowie, P.R., 2011. Plastic contamination in the decapod crustacean Nephrops norvegicus (Linnaeus, 1758). Marine Pollution Bulletin 62, 1207-1217.

Ng, K.L., Obbard, J.P., 2006. Prevalence of microplastics in Singapore's coastal marine environment. Marine Pollution Bulletin 52, 761-767.

O'Brine, T., Thompson, R.C., 2010. Degradation of plastic carrier bags in the marine environment. Marine Pollution Bulletin 60, 2279-2283.

Oehlmann, J.r., Schulte-Oehlmann, U., Kloas, W., Jagnytsch, O., Lutz, I., Kusk, K.O., Wollenberger, L., Santos, E.M., Paull, G.C., Van Look, K.J.W., Tyler, C.R., 2009. A critical analysis of the biological impacts of plasticizers on wildlife. Philosophical Transactions of the Royal Society B: Biological Sciences 364, 2047-2062.

OSPAR, 2007. OSPAR Pilot Project on Monitoring Marine Beach Litter.

Pace, M.L., Bailiff, M.D., 1987. Evaluation of a fluorescent microsphere technique for measuring grazing rates of phagotrophic microorganisms. Marine ecology progress series. Oldendorf. 40, 185-193.

Patel, M.M., Goyal, B.R., Bhadada, S.V., Bhatt, J.S., Amin, A.F., 2009. Getting into the Brain: Approaches to Enhance Brain Drug Delivery, pp. 35-58.

PlasticsEurope, 2010. Plastics - the facts 2010.

Pruter, A.T., 1987. Sources, quantities and distribution of persistent plastics in the marine environment. Marine Pollution Bulletin 18, 305-310.

Rands, M.R.W., Adams, W.M., Bennun, L., Butchart, S.H.M., Clements, A., Coomes, D., Entwistle, A., Hodge, I., Kapos, V., Scharlemann, J.r.P.W., Sutherland, W.J., Vira, B., 2010. Biodiversity Conservation: Challenges Beyond 2010. 329, 1298-1303. 
Richardson, A.J., Walne, A.W., John, A.W.G., Jonas, T.D., Lindley, J.A., Sims, D.W.,

Stevens, D., Witt, M., 2006. Using continuous plankton recorder data. Progress In Oceanography 68, 27-74.

Rios, L.M., Moore, C., Jones, P.R., 2007. Persistent organic pollutants carried by synthetic polymers in the ocean environment. Marine Pollution Bulletin 54, 12301237.

Roy, P.K., Hakkarainen, M., Varma, I.K., Albertsson, A.-C., 2011. Degradable Polyethylene: Fantasy or Reality. Environmental Science \& Technology 45, 42174227.

Ryan, P.G., Moore, C.J., van Franeker, J.A., Moloney, C.L., 2009. Monitoring the abundance of plastic debris in the marine environment. Philosophical Transactions of the Royal Society B: Biological Sciences 364, 1999-2012.

Shaw, D.G., Day, R.H., 1994. Colour- and Form-dependent loss of plastic microdebris from the North Pacific Ocean. Marine Pollution Bulletin 28, 39-43.

Sivan, A., 2011. New perspectives in plastic biodegradation. Current Opinion in Biotechnology 22, 422-426.

Sutherland, W.J., Clout, M., Côté, I.M., Daszak, P., Depledge, M.H., Fellman, L., Fleishman, E., Garthwaite, R., Gibbons, D.W., De Lurio, J., Impey, A.J., Lickorish, F., Lindenmayer, D., Madgwick, J., Margerison, C., Maynard, T., Peck, L.S., Pretty, J., Prior, S., Redford, K.H., Scharlemann, J.P.W., Spalding, M., Watkinson, A.R., 2010. A horizon scan of global conservation issues for 2010. Trends in Ecology \& Evolution $25,1-7$.

Talsness, C.E., Andrade, A.J.M., Kuriyama, S.N., Taylor, J.A., vom Saal, F.S., 2009. Components of plastic: experimental studies in animals and relevance for human 
health. Philosophical Transactions of the Royal Society B: Biological Sciences 364, 2079-2096.

Teuten, E.L., Rowland, S.J., Galloway, T.S., Thompson, R.C., 2007. Potential for Plastics to Transport Hydrophobic Contaminants. Environmental Science \& Technology 41, 7759-7764.

Teuten, E.L., Saquing, J.M., Knappe, D.R.U., Barlaz, M.A., Jonsson, S., BjÃrn, A., Rowland, S.J., Thompson, R.C., Galloway, T.S., Yamashita, R., Ochi, D., Watanuki, Y., Moore, C., Viet, P.H., Tana, T.S., Prudente, M., Boonyatumanond, R., Zakaria, M.P., Akkhavong, K., Ogata, Y., Hirai, H., Iwasa, S., Mizukawa, K., Hagino, Y., Imamura, A., Saha, M., Takada, H., 2009. Transport and release of chemicals from plastics to the environment and to wildlife. Philosophical Transactions of the Royal Society B: Biological Sciences 364, 2027-2045.

Thompson, R., Moore, C., Andrady, A., Gregory, M., Takada, H., Weisberg, S., 2005. New Directions in Plastic Debris, Science, pp. 1117b-.

Thompson, R.C., 2006. Plastic debris in the marine environment: consequences and solutions, in: Krause, J.C., Nordheim, H., Bräger, S. (Eds.), Marine Nature Conservation in Europe. Federal Agency for Nature Conservation, Stralsund, Germany, pp. 107-115.

Thompson, R.C., Moore, C.J., vom Saal, F.S., Swan, S.H., 2009a. Plastics, the environment and human health: current consensus and future trends. Philosophical Transactions of the Royal Society B: Biological Sciences 364, 2153-2166.

Thompson, R.C., Olsen, Y., Mitchell, R.P., Davis, A., Rowland, S.J., John, A.W.G., McGonigle, D., Russell, A.E., 2004. Lost at Sea: Where Is All the Plastic?, Science, p. 838. 
Thompson, R.C., Swan, S.H., Moore, C.J., vom Saal, F.S., 2009b. Our plastic age. Philosophical Transactions of the Royal Society B: Biological Sciences 364, 19731976.

Tourinho, P.S., Ivar do Sul, J.A., Fillmann, G., 2010. Is marine debris ingestion still a problem for the coastal marine biota of southern Brazil? Marine Pollution Bulletin 60, 396-401.

Turner, A., Holmes, L., 2011. Occurrence, distribution and characteristics of beached plastic production pellets on the island of Malta (central Mediterranean). Marine Pollution Bulletin 62, 377-381.

van Franeker, J.A., 2010. Fulmar Litter EcoQO Monitoring in the Netherlands 19792008 in relation to EU Directive 2000/59/EC on Port Reception Facilities. IMARES Wageningen UR.

van Franeker, J.A., Blaize, C., Danielsen, J., Fairclough, K., Gollan, J., Guse, N., Hansen, P.-L., Heubeck, M., Jensen, J.-K., Le Guillou, G., Olsen, B., Olsen, K.-O., Pedersen, J., Stienen, E.W.M., Turner, D.M., 2011. Monitoring plastic ingestion by the northern fulmar Fulmarus glacialis in the North Sea. Environmental Pollution 159, 2609-2615.

vom Saal, F.S., Myers, J.P., 2008. Bisphenol A and Risk of Metabolic Disorders. Journal of the American Medical Association 300, 1353-1355.

Watters, D.L., Yoklavich, M.M., Love, M.S., Schroeder, D.M., 2010. Assessing marine debris in deep seafloor habitats off California. Marine Pollution Bulletin 60, $131-138$

Wilson, D.S., 1973. Food Size Selection Among Copepods. Ecology 54, 909-914. 
Winston, J.E., 1982. Drift Plastic - An expanding niche for a marine invertebrate. Marine Pollution Bulletin 13, 348-351.

Zarfl, C., Fleet, D., Fries, E., Galgani, F., Gerdts, G., Hanke, G., Matthies, M., 2011. Microplastics in oceans. Marine Pollution Bulletin 62, 1589-1591.

Zarfl, C., Matthies, M., 2010. Are marine plastic particles transport vectors for organic pollutants to the Arctic? Marine Pollution Bulletin 60, 1810-1814.

Zitko, V., Hanlon, M., 1991. Another Source of Pollution by Plastics: Skin Cleansers with Plastic Scrubbers. Marine Pollution Bulletin 22, 41-42. 